International Mathematical Forum, Vol. 8, 2013, no. 8, 369 - 375

\title{
Inference Methods for Stochastic Volatility Models
}

\author{
Maddalena Cavicchioli
}

Cá Foscari University of Venice

Advanced School of Economics

Cannaregio 231, Venice, Italy

maddalena.cavicchioli@unive.it

\begin{abstract}
In the present paper we consider estimation procedures for stationary Stochastic Volatility models, making inferences about the latent volatility of the process. We show that a sequence of generalized least squares regressions enables us to determine the estimates. Finally, we make inferences iteratively by using the Kalman Filter algorithm.
\end{abstract}

Mathematics Subject Classification: 62M10, 62M20, 91B84, 93E03

Keywords: Stochastic Volatility, Generalized Least Squares, Kalman Filter

\section{Introduction}

Stochastic Volatility (SV) models have been received a growing interest in time series analysis since they find many financial applications as, for example, option pricing, asset allocation and risk management. For a comprehensive discussion on SV models see, for example, Taylor [3] and Tsyplakov [4]. Let us consider the basic stochastic volatility model given by

$$
\begin{aligned}
y_{t} & =\exp \left\{\frac{1}{2} h_{t}(\theta)\right\} u_{t} \\
h_{t}(\theta) & =\mu+\rho h_{t-1}(\theta)+v_{t}
\end{aligned}
$$

where the error terms $u_{t} \sim \operatorname{IIN}(0,1)$ and $v_{t} \sim \operatorname{IIN}\left(0, \sigma_{v}^{2}\right)$ are assumed to be independent of one other, and the parameter vector $\theta=\left(\mu \rho \sigma_{v}^{2}\right)$ is in a compact parameter set $\Theta \subset(0,+\infty)^{3}$. To ensure stationarity, we always set $|\rho|<1$. In this case we have

$$
h_{t}(\theta)=\mu(1-\rho)^{-1}+\sum_{i=0}^{\infty} \rho^{i} v_{t-i}
$$


hence $E\left(h_{t}\right)=\mu(1-\rho)^{-1}$ and $\operatorname{Var}\left(h_{t}\right)=\sigma_{v}^{2}\left(1-\rho^{2}\right)^{-1}$. Squaring (1.1) and taking logs, we get the state-space representation

$$
\begin{aligned}
x_{t} & =\alpha+h_{t}(\theta)+e_{t} \\
h_{t}(\theta) & =\mu+\rho h_{t-1}(\theta)+v_{t}
\end{aligned}
$$

where $x_{t}=\log y_{t}^{2}, \alpha=E\left[\log u_{t}^{2}\right]$ is a real constant $(\simeq-1.27)$ and $e_{t}=\log u_{t}^{2}-\alpha$ is a martingale difference but not normal, i.e., $e_{t} \sim \operatorname{IID}\left(0, \sigma_{e}^{2}\right)$, where $\sigma_{e}^{2}=$ $\pi^{2} / 2$ (here $\log$ denotes the natural logarithm). See Breidt and Carriquiry [1]. Following Kim-Nelson [2], Chp.3, we present two alternative ways of making inferences about $h_{t}$ (volatility) conditional on information available up to time $t$ (given $\theta$ ). In Section 2 we show that a sequence of generalized least squares (GLS) regressions enables us to determine $h_{t \mid t}=E\left(h_{t} \mid \Psi_{t}\right)$, where $\Psi_{t}$ denotes the information set up to time $t$. In Section 3 we make inferences about $h_{t}$ by employing the Kalman Filter algorithm.

\section{Generalized Least Squares Estimation}

As usual, we approximates the SV model in (1.3) by a Gaussian state-space model. From (1.3) we get

$$
h_{t}=\mu(1-\rho)^{-1}\left(1-\rho^{t-1}\right)+\rho^{t-1} h_{1}+\sum_{i=0}^{t-2} \rho^{i} v_{t-i}
$$

for $t \geq 2$. Thus

$$
\left[\begin{array}{c}
h_{1} \\
h_{2} \\
\vdots \\
h_{t-1} \\
h_{t}
\end{array}\right]=\left[\begin{array}{c}
\rho^{-t+1} \\
\rho^{-t+2} \\
\vdots \\
\rho^{-1} \\
1
\end{array}\right] h_{t}-\left[\begin{array}{c}
\rho^{-1} v_{2}+\rho^{-2} v_{3}+\cdots+\rho^{-t+2} v_{t-1}+\rho^{-t+1} v_{t} \\
\rho^{-1} v_{3}+\cdots+\rho^{-t+3} v_{t-1}+\rho^{-t+2} v_{t} \\
\vdots \\
\rho^{-1} v_{t} \\
0
\end{array}\right]-\mu\left[\begin{array}{c}
\frac{\rho^{-t+1}-1}{1-\rho} \\
\frac{\rho^{-t+2}-1}{1-\rho} \\
\vdots \\
\rho^{-1} \\
0
\end{array}\right]
$$

Define:

$$
\mathbf{a}_{t}=\left(\begin{array}{lllll}
\rho^{-t+1} & \rho^{-t+2} & \ldots & \rho^{-1} & 1
\end{array}\right)^{\prime}
$$

and

$$
B_{t}=\left[\begin{array}{ccccc}
\rho^{-1} & \rho^{-2} & \ldots & \rho^{-t+2} & \rho^{-t+1} \\
0 & \rho^{-1} & \ldots & \rho^{-t+3} & \rho^{-t+2} \\
\vdots & \vdots & & \vdots & \vdots \\
0 & 0 & \ldots & 0 & \rho^{-1} \\
0 & 0 & \ldots & 0 & 0
\end{array}\right] \quad C_{t}=\left[\begin{array}{c}
\alpha-\mu(1-\rho)^{-1}\left(\rho^{-t+1}-1\right) \\
\alpha-\mu(1-\rho)^{-1}\left(\rho^{-t+2}-1\right) \\
\vdots \\
\alpha-\mu \rho^{-1} \\
\alpha
\end{array}\right]
$$


Using the measurement equation in (1.3) and the above matrix relation, we have

$$
\mathbf{x}_{t}=C_{t}+\mathbf{a}_{t} h_{t}+\boldsymbol{\epsilon}_{t}
$$

where $\mathbf{x}_{t}=\left(x_{1} x_{2} \ldots x_{t}\right)^{\prime}, \boldsymbol{\epsilon}_{t}=-B_{t}\left(v_{2} \ldots v_{t}\right)^{\prime}+\mathbf{e}_{t}$ and $\mathbf{e}_{t}=\left(e_{1} e_{2} \ldots e_{t}\right)^{\prime}$. Then we have

$$
E\left(\boldsymbol{\epsilon}_{t} \boldsymbol{\epsilon}_{t}^{\prime}\right)=\sigma_{e}^{2} I_{t}+\sigma_{v}^{2} B_{t} B_{t}^{\prime}=\Omega_{t} .
$$

One could apply GLS to model $(2.2)$ for $t=2, \ldots, T$. Then we get

$$
h_{t \mid t}=\left(\mathbf{a}_{t}^{\prime} \Omega_{t}^{-1} \mathbf{a}_{t}\right)^{-1} \mathbf{a}_{t}^{\prime} \Omega_{t}^{-1}\left(\mathbf{x}_{t}-C_{t}\right)
$$

hence

$$
h_{t \mid t}=h_{t}+\left(\mathbf{a}_{t}^{\prime} \Omega_{t}^{-1} \mathbf{a}_{t}\right)^{-1} \mathbf{a}_{t}^{\prime} \Omega_{t}^{-1} \boldsymbol{\epsilon}_{t} .
$$

Then we have

$$
E\left(h_{t \mid t}\right)=E\left(h_{t}\right)=\mu(1-\rho)^{-1}
$$

and

$$
P_{t \mid t}=E\left[h_{t}-h_{t \mid t}\right]^{2}=\left(\mathbf{a}_{t}^{\prime} \Omega_{t}^{-1} \mathbf{a}_{t}\right)^{-1} .
$$

Define

$$
\mathbf{b}_{t}=B_{t}^{\prime} \mathbf{a}_{t}\left\|\mathbf{a}_{t}\right\|^{-2}=\frac{1}{1-\rho^{-2 t}}\left[\begin{array}{c}
\rho^{-t}\left(1-\rho^{-2}\right) \\
\rho^{-t+1}\left(1-\rho^{-4}\right) \\
\vdots \\
\rho^{-2}\left(1-\rho^{-2 t+2}\right)
\end{array}\right] .
$$

Theorem 2.1 With the above notation, we have

$$
P_{t \mid t}=\sigma_{e}^{2}\left\|\mathbf{a}_{t}\right\|^{-2}+\sigma_{v}^{2}\left\|\mathbf{b}_{t}\right\|^{2}
$$

where

$$
\left\|\mathbf{a}_{t}\right\|^{2}=\frac{1-\rho^{-2 t}}{1-\rho^{-2}} \quad\left\|\mathbf{b}_{t}\right\|^{2}=\frac{\rho^{-4 t+2}-1}{\rho^{2}\left(1-\rho^{2}\right)\left(1-\rho^{-2 t}\right)^{2}}-\frac{2 t-1}{\rho^{2 t+2}\left(1-\rho^{-2 t}\right)^{2}} .
$$

Proof. We apply the Sherman-Morrison-Woodbury (SMW) formula, i.e., if $A$ and $C$ are invertible matrices, then

$$
(A+B C D)^{-1}=A^{-1}-A^{-1} B\left(C^{-1}+D A^{-1} B\right)^{-1} D A^{-1} .
$$

Setting $A=\sigma_{e}^{2} I_{t}, B=B_{t}, C=\sigma_{v}^{2} I_{t-1}$ and $D=B_{t}^{\prime}$, from (2.3) we get

$$
\Omega_{t}^{-1}=\sigma_{e}^{-2} I_{t}-\sigma_{e}^{-2} B_{t}\left(\sigma_{v}^{-2} I_{t-1}+\sigma_{e}^{-2} B_{t}^{\prime} B_{t}\right)^{-1} \sigma_{e}^{-2} B_{t}^{\prime}
$$


hence

$$
\mathbf{a}_{t}^{\prime} \Omega_{t}^{-1} \mathbf{a}_{t}=\sigma_{e}^{-2} \mathbf{a}_{t}^{\prime} \mathbf{a}_{t}-\sigma_{e}^{-2} \mathbf{a}_{t}^{\prime} B_{t}\left(\sigma_{v}^{-2} I_{t-1}+\sigma_{e}^{-2} B_{t}^{\prime} B_{t}\right)^{-1} \sigma_{e}^{-2} B_{t}^{\prime} \mathbf{a}_{t} .
$$

Apply again the SMW formula with $A=\sigma_{e}^{-2} \mathbf{a}_{t}^{\prime} \mathbf{a}_{t}, B=-\sigma_{e}^{-2} \mathbf{a}_{t}^{\prime} B_{t}, C=$ $\left(\sigma_{v}^{-2} I_{t-1}+\sigma_{e}^{-2} B_{t}^{\prime} B_{t}\right)^{-1}$ and $D=\sigma_{e}^{-2} B_{t}^{\prime} \mathbf{a}_{t}$. Then we have

$$
\begin{aligned}
P_{t \mid t}= & \left(\mathbf{a}_{t}^{\prime} \Omega_{t}^{-1} \mathbf{a}_{t}\right)^{-1}=\sigma_{e}^{2}\left(\mathbf{a}_{t}^{\prime} \mathbf{a}_{t}\right)^{-1}-\left(\mathbf{a}_{t}^{\prime} \mathbf{a}_{t}\right)^{-1} \mathbf{a}_{t}^{\prime} B_{t} \\
& \left(\sigma_{v}^{-2} I_{t-1}+\sigma_{e}^{-2} B_{t}^{\prime} B_{t}-\sigma_{e}^{-2} B_{t}^{\prime} \mathbf{a}_{t}\left(\mathbf{a}_{t}^{\prime} \mathbf{a}_{t}\right)^{-1} \mathbf{a}_{t}^{\prime} B_{t}\right)^{-1} B_{t}^{\prime} \mathbf{a}_{t}\left(\mathbf{a}_{t}^{\prime} \mathbf{a}_{t}\right)^{-1} \\
= & \sigma_{e}^{2}\left\|\mathbf{a}_{t}\right\|^{-2}+\left(\mathbf{a}_{t}^{\prime} \mathbf{a}_{t}\right)^{-1} \mathbf{a}_{t}^{\prime} B_{t}\left(\sigma_{v}^{-2} I_{t-1}\right)^{-1} B_{t}^{\prime} \mathbf{a}_{t}\left(\mathbf{a}_{t}^{\prime} \mathbf{a}_{t}\right)^{-1} \\
= & \sigma_{e}^{2}\left\|\mathbf{a}_{t}\right\|^{-2}+\sigma_{v}^{2}\left\|\mathbf{b}_{t}\right\|^{2}
\end{aligned}
$$

which gives the result of the statement.

\section{Theorem 2.2}

$$
P_{\infty}=\lim _{T \rightarrow+\infty} \frac{1}{T} \sum_{t=1}^{T} P_{t \mid t}=\sigma_{v}^{2}\left(1-\rho^{2}\right)^{-1}=\operatorname{var}\left(h_{t}\right) .
$$

Proof. To compute the partial sums of the series we took advantage of the software "Mathematica". We have (recall that $|\rho|<1$ )

$$
\sum_{t=1}^{T}\left\|\mathbf{a}_{t}\right\|^{-2}=\sum_{t=1}^{T} \frac{1-\rho^{-2}}{1-\rho^{-2 t}}=\frac{\left(\rho^{2}-1\right) \psi_{\rho^{2}}^{(0)}(T+1)+\left(1-\rho^{2}\right) \psi_{\rho^{2}}^{(0)}(1)}{\rho^{2} \log \left(\rho^{2}\right)}
$$

where $\psi_{q}^{(0)}(z)=\psi_{q}(z)=\partial \log \Gamma_{q}(z) / \partial z$ denotes the $q$-digamma function. Since

$$
\lim _{T \rightarrow+\infty} \frac{1}{T} \psi_{\rho^{2}}^{(0)}(T+1)=0
$$

we get

$$
\lim _{T \rightarrow+\infty} \frac{1}{T} \sum_{t=1}^{T}\left\|\mathbf{a}_{t}\right\|^{-2}=0
$$

Furthermore, we have

$$
\sum_{t=1}^{T} \frac{\rho^{-4 t+2}-1}{\rho^{2}\left(1-\rho^{2}\right)\left(1-\rho^{-2 t}\right)^{2}}=\frac{\psi_{\rho^{2}}^{(1)}(T+1)}{\rho^{2} \log ^{2}\left(\rho^{2}\right)}+\frac{\left(\rho^{2}+1\right) \psi_{\rho^{2}}^{(0)}(T+1)}{\rho^{2}\left(\rho^{2}-1\right) \log \left(\rho^{2}\right)}+\frac{T}{1-\rho^{2}}+c
$$

where $c$ is the numerical constant

$$
c=-\frac{\psi_{\rho^{2}}^{(1)}(1)}{\rho^{2} \log ^{2}\left(\rho^{2}\right)}-\frac{\left(\rho^{2}+1\right) \psi_{\rho^{2}}^{(0)}(1)}{\rho^{2}\left(\rho^{2}-1\right) \log \left(\rho^{2}\right)}
$$


and $\psi_{q}^{(1)}(z)$ denotes the first derivative of the $q$-digamma function. Now (2.6) and

$$
\lim _{T \rightarrow+\infty} \frac{1}{T} \psi_{\rho^{2}}^{(1)}(T+1)=0
$$

imply

$$
\lim _{T \rightarrow+\infty} \frac{1}{T} \sum_{t=1}^{T} \frac{\rho^{-4 t+2}-1}{\rho^{2}\left(1-\rho^{2}\right)\left(1-\rho^{-2 t}\right)^{2}}=\frac{1}{1-\rho^{2}}
$$

It remains to consider the series

$$
\sum_{t=1}^{T} \frac{2 t-1}{\rho^{2 t+2}\left(1-\rho^{-2 t}\right)^{2}}=\sum_{t=1}^{T} \frac{2 t-1}{\rho^{2}\left(\rho^{t}-\rho^{-t}\right)^{2}} .
$$

We take the first Taylor expansion around $\rho_{0} \in(0,1)$ of the function $\left(\rho^{t}-\rho^{-t}\right)^{2}$ and use the following sequence of inequalities

$$
\begin{aligned}
0<\frac{2 t-1}{\left(\rho^{t}-\rho^{-t}\right)^{2}} & \sim \frac{2 t-1}{\left(\rho_{0}^{t}-\rho_{0}^{-t}\right)^{2}+2 t\left(\rho_{0}^{t}-\rho_{0}^{-t}\right)\left(\rho_{0}^{t-1}+\rho_{0}^{-t-1}\right)\left(\rho-\rho_{0}\right)} \\
& <\frac{1}{\left(\rho_{0}^{t}-\rho_{0}^{-t}\right)\left(\rho_{0}^{t-1}+\rho_{0}^{-t-1}\right)\left(\rho-\rho_{0}\right)}
\end{aligned}
$$

for $0<\rho<\rho_{0}$. But we have

$$
\begin{array}{r}
\sum_{t=1}^{T} \frac{1}{\left(\rho^{t}-\rho^{-t}\right)\left(\rho^{t-1}+\rho^{-t-1}\right)}=\frac{\rho \psi_{\rho}^{(0)}(T+1)-\rho \psi_{\rho}^{(0)}\left(T-\frac{i \pi}{2 \log (\rho)}+1\right)}{4 \log (\rho)} \\
+\frac{-\rho \psi_{\rho}^{(0)}\left(T+\frac{i \pi}{2 \log (\rho)}+1\right)+\rho \psi_{\rho}^{(0)}\left(T-\frac{i \pi}{\log (\rho)}+1\right)}{4 \log (\rho)}+d
\end{array}
$$

where $d$ is the numerical constant

$$
d=\frac{-\rho \psi_{\rho}^{(0)}(1)+\rho \psi_{\rho}^{(0)}\left(1-\frac{i \pi}{2 \log (\rho)}\right)+\rho \psi_{\rho}^{(0)}\left(1+\frac{i \pi}{2 \log (\rho)}\right)-\rho \psi_{\rho}^{(0)}\left(1-\frac{i \pi}{2 \log (\rho)}\right)}{4 \log (\rho)}
$$

Then we have

$$
\lim _{T \rightarrow+\infty} \frac{1}{T} \sum_{t=1}^{T} \frac{1}{\left(\rho^{t}-\rho^{-t}\right)\left(\rho^{t-1}+\rho^{-t-1}\right)}=0
$$

as

$$
\begin{aligned}
\lim _{T \rightarrow+\infty} \frac{1}{T} \psi_{\rho}^{(0)}(T+1) & =\lim _{T \rightarrow+\infty} \frac{1}{T} \psi_{\rho}^{(0)}\left(T \pm \frac{i \pi}{2 \log (\rho)}+1\right) \\
& =\lim _{T \rightarrow+\infty} \frac{1}{T} \psi_{\rho}^{(0)}\left(T-\frac{i \pi}{\log (\rho)}+1\right)=0 .
\end{aligned}
$$


This implies

$$
\lim _{T \rightarrow+\infty} \frac{1}{T} \sum_{t=1}^{T} \frac{2 t-1}{\rho^{2}\left(\rho^{t}-\rho^{-t}\right)^{2}}=0
$$

hence

$$
\lim _{T \rightarrow+\infty} \frac{1}{T} \sum_{t=1}^{T}\left\|\mathbf{b}_{t}\right\|^{2}=\frac{1}{1-\rho^{2}}
$$

Finally, from (2.7) and (2.10), we get the result of the statement.

\section{Estimation by the Kalman Filter}

Since Model (1.3) is in linear state-space form, predicted filtered and smoothed values of $h_{t}$ can be computed recursively via the Kalman Filter algorithm. See Kim-Nelson [2], Sec.3.1.2. Define $h_{t \mid \tau}=E\left[h_{t} \mid \Psi_{\tau}\right], P_{t \mid \tau}=E\left[h_{t}-h_{t \mid \tau}\right]^{2}$, $x_{t \mid \tau}=E\left[x_{t} \mid \Psi_{\tau}\right], \eta_{t \mid \tau}=x_{t}-x_{t \mid \tau}$ and $f_{t \mid \tau}=E\left[\eta_{t \mid \tau}\right]^{2}$ for $1 \leq \tau \leq T$. For the one-step-ahead prediction, we have

$$
\begin{aligned}
& h_{t \mid t-1}=\mu+\rho h_{t-1 \mid t-1} \quad P_{t \mid t-1}=\rho^{2} P_{t-1 \mid t-1}+\sigma_{v}^{2} \\
& \eta_{t \mid t-1}=x_{t}-x_{t \mid t-1}=x_{t}-\alpha-h_{t \mid t-1}=\left(h_{t}-h_{t \mid t-1}\right)+e_{t} \\
& f_{t \mid t-1}=P_{t \mid t-1}+\sigma_{e}^{2} .
\end{aligned}
$$

The initial states of the recursion are

$$
h_{0 \mid 0}=\mu(1-\rho)^{-1} \quad \text { and } \quad P_{0 \mid 0}=\sigma_{v}^{2}\left(1-\rho^{2}\right)^{-1} .
$$

The updating is given by

$$
\begin{aligned}
& h_{t \mid t}=h_{t \mid t-1}+K_{t} \eta_{t \mid t-1}=h_{t \mid t-1}+K_{t}\left(x_{t}-\alpha-h_{t \mid t-1}\right) \\
& P_{t \mid t}=P_{t \mid t-1}-K_{t} P_{t \mid t-1}=P_{t \mid t-1}-P_{t \mid t-1}^{2} f_{t \mid t-1}^{-1}
\end{aligned}
$$

where

$$
K_{t}=P_{t \mid t-1} f_{t \mid t-1}^{-1}=P_{t \mid t-1}\left(P_{t \mid t-1}+\sigma_{e}^{2}\right)^{-1}
$$

is the Kalman gain. From these recursions, one can construct the (quasi) Gaussian log-likelihood

$$
\ell\left(\theta \mid \Psi_{T}\right)=-\frac{T}{2} \log (2 \pi)-\frac{1}{2} \sum_{t=1}^{T} \log f_{t \mid t-1}-\frac{1}{2} \sum_{t=1}^{T} \eta_{t \mid t-1}^{2} f_{t \mid t-1}^{-1} .
$$

The smoothed estimates and their variances are given by

$$
\begin{aligned}
& h_{t \mid T}=h_{t \mid t}+P_{t}^{*}\left(h_{t+1 \mid T}-\mu-\rho h_{t \mid t}\right) \\
& P_{t \mid T}=P_{t \mid t}+\left[P_{t}^{*}\right]^{2}\left(P_{t+1 \mid T}-P_{t+1 \mid t}\right)
\end{aligned}
$$


where

$$
P_{t}^{*}=\rho P_{t \mid t} P_{t+1 \mid t}^{-1}
$$

Solving the difference equations in $h_{t \mid t}$ and $P_{t \mid t}$ gives the estimates. More precisely, we have

$$
\begin{aligned}
& P_{t \mid t}=\sigma_{v}^{2} \rho^{2 t}\left(1-\rho^{2}\right)^{-1} \prod_{i=0}^{t-1}\left(1-K_{t-i}\right)+\sigma_{v}^{2} \sum_{k=0}^{t-1} \rho^{2 k} \prod_{j=0}^{k}\left(1-K_{t-j}\right) \\
& h_{t \mid t}=\mu(1-\rho)^{-1}+\sum_{i=0}^{\infty} \rho^{i} K_{t-i} \eta_{t-i \mid t-i-1} \\
& \eta_{t \mid t-1}=\alpha_{t}(L) v_{t}+\beta_{t}(L) e_{t}
\end{aligned}
$$

where $\alpha_{t}(L)=1+\sum_{r=1}^{t} \rho^{r} \prod_{s=1}^{r}\left(1-K_{t-s}\right) L^{r}$ and $\beta_{t}(L)=\alpha_{t}(L)(1-\rho L)$ (here $L$ denotes the lag operator).

\section{References}

[1] F.J. Breidt and A.L. Carriquiry, Quasi-Maximum Likelihood Estimation for Stochastic Volatility Models, in Modelling and Prediction, Honoring Seymour Geisel (A. Zellner, J.S. Lee, eds.), Springer Verlag, (1996).

[2] C.J. Kim and C.R. Nelson, State-Space Models with Regime Switching. Classical and Gibbs-Sampling Approaches with Applications, The MIT Press, Cambridge. MA, (1999).

[3] S.J. Taylor, Modelling Stochastic Volatility, Mathematical Finance, 4 (1994), $183-204$.

[4] A. Tsyplakov, Revealing the arcane: an introduction to the art of Stochastic Volatility models, MPRA, no.25511, 2010.

\section{Received: November, 2012}

